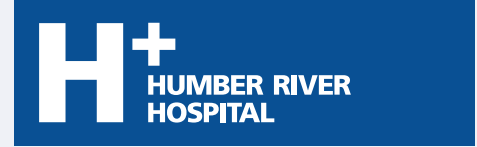

HUMBER RIVER HOSPITAL is one of Canada's largest community acute care hospitals, serving a population of more than 850,000 people in the northwest Greater Toronto Area.

The multi-site hospital currently operates out of its Wilson Avenue acute care site and Finch and Church Street reactivation care centres with a total of 722 beds, just over 3,800 employees, approximately 700 physicians and over 1,000 volunteers.

Affiliated with the University of Toronto and Queen's University, Humber River Hospital is North America's first fully digital hospital. Part of Humber River Hospital's digital infrastructure includes completely automated laboratory services, robots sorting and mixing medications, electronic health records, tracking systems for patients undergoing surgery that update families through their cellphones and patient computer bedside terminals - all varieties of technologies that automate information, eliminate paper and provide a connected experience for patients, staff and families.

Humber River Hospital was awarded Accreditation with Exemplary Standing in 2018 and since its opening in 2015 has received numerous awards and accolades for technological advancements and innovation (www.hrh.ca).

\title{
Smartphone Technology: Enabling Prioritization of Patient Needs and Enhancing the Nurse-Patient Relationship
}

Vanessa Burkoski, RN, BScN, MScN, DHA Chief Nursing Executive \& Chief, People Strategy

Humber River Hospital

Toronto, ON

Jennifer Yoon, RN, BScN, MSc(QI/PS)

Senior Director

Professional Practice, Quality \&

Patient Safety

Humber River Hospital

Toronto, ON

Derek Hutchinson, RN, BScN, MN Director, Professional Practice

Humber River Hospital

Toronto, ON
Kevin Fernandes, C.Tech, ITIL Chief Technology Officer Information Technology \& Telecom

Humber River Hospital

Toronto, ON

Shirley Solomon, BA, MSc

Research Assistant

Humber River Hospital

Toronto, ON

Barbara E. Collins, RN, BScN, MBA

President \& CEO

Humber River Hospital

Toronto, ON

Scott R. Jarrett, BSc, MBA, MLT

Executive Vice President \&

Chief of Clinical Programs

Humber River Hospital

Toronto, ON 


\section{WHAT WE'VE LEARNED:}

1. There are multiple benefits of smartphone technology, including time management, convenience, prioritization, patient safety and enhancement of the nurse-patient relationship. Issues such as patients' lack of knowledge regarding how to use the call system, insufficient sound quality and poor battery life are challenges with the smartphone that need to be addressed.
2. Hospitals proposing to introduce smartphone technology need to educate patients and families about the clinical use and functionality of communication information technology to avoid unfavourable perceptions.
3. Smartphone technology must be integrated with other health information systems and requires interoperability with the EMR to maximize interprofessional communication and exchange of patient information.

\section{Abstract}

Background: Mobile health (mHealth) is a rapidly growing field with the potential to transform healthcare delivery. Smartphone technologies have been developed and integrated into the patient call bell system for healthcare staff to receive calls; however, there is a lack of high-quality evidence to support the implementation and evaluate the effectiveness of these devices in a healthcare setting.

Aim: The aim of this study is to explore nurses' perceptions of smartphone technology devices in enhancing the nurse-patient relationship and improving nursing workflows.

Methods: A semi-structured focus group and interviews were used to illicit nurses' experiences with smartphone technology. Interviews were audio recorded, transcribed and subjected to a content analysis to identify emerging themes from the data.

Results: Interviews with nurses provided insight into the benefits and challenges of smartphone use in the clinical setting. Multiple benefits were identified by nurse participants, including time management and convenience, prioritization, patient safety and enhancement of the nurse-patient relationship.

Conclusion: There are multiple benefits of smartphone technology for both nurses and patients. Hospitals proposing to introduce smartphone technology need to educate patients and families about the clinical use of smartphones to avoid unfavourable perceptions. Smartphone technology must be interoperable with the electronic medical record to optimize interprofessional communication and exchange of patient information. 
Mobile technology has evolved from a personal device to a clinical tool with the potential to revolutionize healthcare delivery. Humber River Hospital (HRH) has taken a technological leap by integrating smartphones into the patient call bell system to further support nurses' communication. The new system aims to improve nurses' ability to manage patient calls and prioritize patient needs (Figure 1).

\section{Figure 1.}

The various components of the nurse call system (ways patients can contact the nurse).

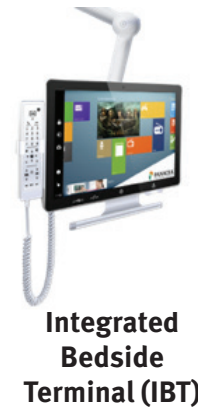

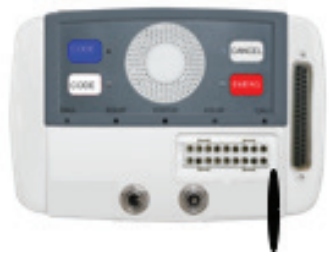

Patient Station

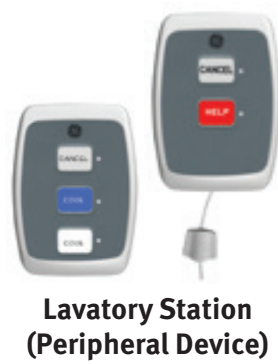

(B)

Patient Call Cords (Peripheral Device)
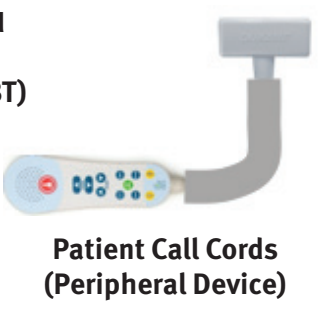
A review of published and grey literature evaluating the impact of mobile health (mHealth) on communications between staff has demonstrated that mobile technology (see Figure 2) has the ability to reduce overhead paging noise and positively impact nursing workflow and staff ability to provide safe, high-quality care (Doswell et al. 2013; Whitlow et al. 2014). The aim of this study was to examine the potential of smartphone technology to improve nursing workflows and enhance the nurse-patient relationship at HRH.

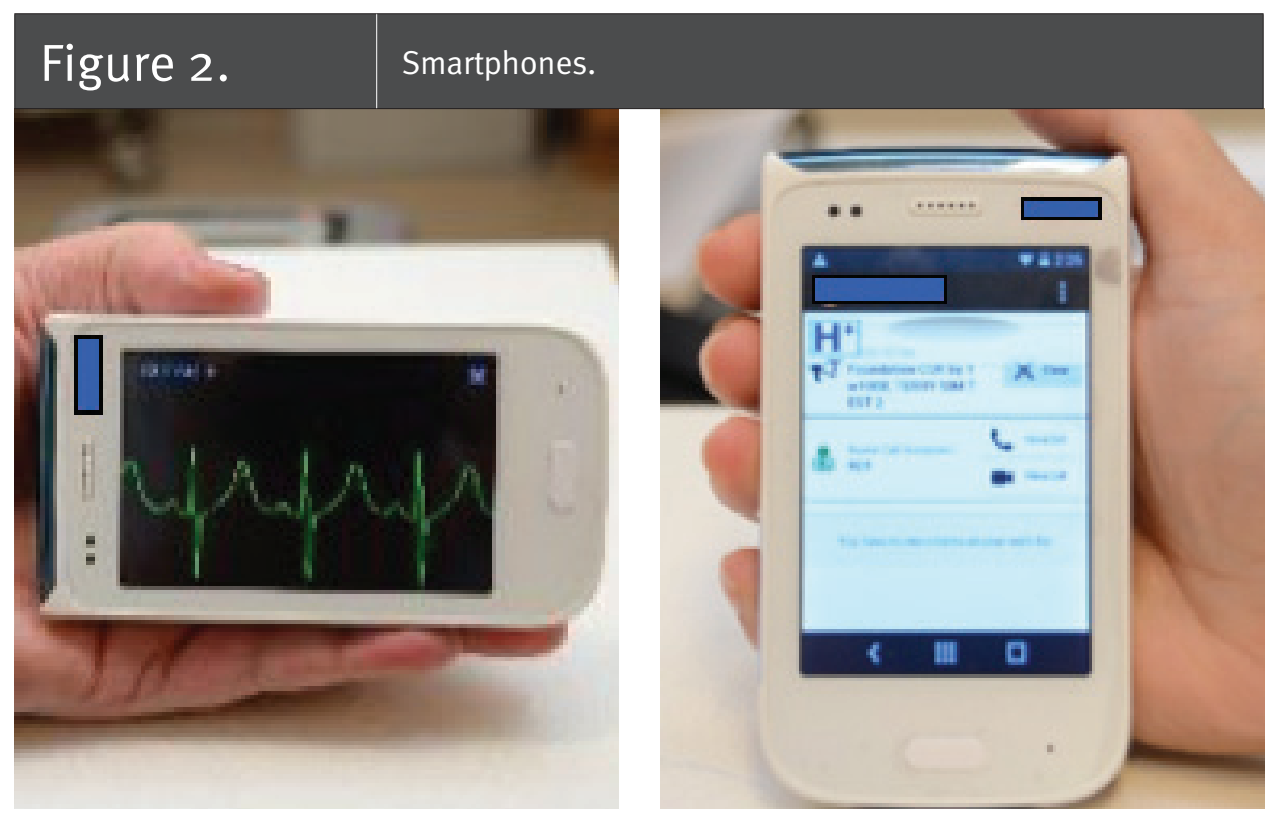

\section{Methods}

Study sample and data collection

A qualitative exploratory study was employed to investigate nurses' perceptions of smartphone technology devices in enabling prioritization of patient needs and enhancing the nurse-patient relationship. Selection criteria included the requirement that nurses predominantly provide direct care to inpatients and have at least six months' experience working at the hospital. Research board ethics approval was obtained. Participation in the study was voluntary, and participants were assured of anonymity.

Qualitative approaches were used to explore nurses' perceptions of hospital smartphone technology. In-depth interviews took place in November 2018 in a private room within the hospital setting and were conducted using a semi-structured format. Open-ended interviewing was used to allow participants to supply information unique to their experiences regarding the use of mobile technology within clinical settings. The interviews lasted between 20 and 40 minutes and were 
digitally recorded. Observation notes were recorded by the interviewer to document participant expressions and body language. Probing questions were asked by the interviewer to further explore points of interest. Participants were encouraged to express their views freely.

Data analysis

Digital recordings were transcribed and subjected to a content analysis. Transcripts were analyzed to identify perceptions that were common across all participants. Quotations were chosen to illustrate emerging themes from the data.

\section{Results}

Of the 12 nurses interviewed, $66.7 \%$ were female and the age range for participants was from 20s to 40s. The average number of years of experience for all participants was 3.79 years $(S D=2.66)$, with 3.11 years $(S D=2.84)$ of employment at HRH. The majority of participants had academic preparation at the level of a baccalaureate degree (83.3\%) and practised as registered nurses (83.3\%).

Time management and convenience

Many of the nurses in this study perceived significant improvements in efficiency over traditional patient call bell systems. The smartphone rings directly to the specific nurse assigned to care for the patient, thereby eliminating the need for the nurse to attend the nursing station to receive information regarding the needs of the patient calling for assistance. This meant that nurses could receive a call from another patient while caring for a patient or involved in a task without having to cease caring for the patient or performing the task at hand. More than half of the nurses from the study stated that the smartphone helped them avoid any unnecessary interruptions:

We didn't have a [smart] phone [in my previous place of employment], so we had to keep checking the call bell. So this has been a very big change.

Before, you'd always have to go to the nursing station when a call came in just to find out who's calling.

At night, if nobody is sitting at the nursing station, nobody is going to know that the patient is calling, or [the traditional call bell] is just so loud that it wakes up all the patients.

Most of the study participants emphasized that having a smartphone was essential because of the magnitude of the physical space of the hospital. One study participant mentioned that if the nursing station is not staffed, it may take a very long time for the patient's call to be answered, and having the smartphone circumvents 
this issue. The extensive walking distance between patient rooms, the nursing station and supply rooms was perceived by nurses as necessitating the appropriate tools, such as the smartphone, to manage time efficiently and effectively. Another advantage of the smartphone was convenience, as one nurse in the interview stated:

The fact [is] that [smartphones] get a call bell to you right away and lab results, and if you have to get a call from a doctor and it goes right to your phone, it's very useful.

With the phone system, any nurse can be easily reached and any critical laboratory results concerning the patient can be delivered directly to the patient's nurse. All of the nurses in the study stated that the smartphone was most beneficial for immediate communication with the interprofessional clinical team, as well as portering services. With the portering service, any nurse will receive a notification by smartphone 15 minutes prior to the patient being transported, allowing nurses additional time to ensure that the patient is prepared for transport when the porter arrives. Some nurses described having the smartphone as a lifeline. One nurse stated,

I almost don't want to go to [work at] another hospital because of the smartphone. It's so useful.

\section{Prioritization}

The smartphone featured a different tone for call bells, alarms activated from the patient's bathroom and alarms activated when patients at high risk for falls exit the bed. Most of the nurses from this study stated that they immediately prioritized bed alarms and bathroom alarms because they presented a safety risk to those patients. Additionally, if a nurse received a call bell from a "high-falls-risk" patient, nurses from this study stated that they would prioritize those requests, knowing that the patient might be requesting help to get up to use the bathroom and would try to do so alone if the nurse did not come promptly.

\section{Patient safety}

Most of the study participants believed that the smartphones were crucial to maintaining patient safety. As previously described, when a high-falls-risk patient exits the bed, an alarm is sent to the nurse's phone:

Some patients try to get up by themselves, or they don't want to wait for a nurse to come to them because they think it will take a while. So we get an alert immediately when a [high falls risk patient] gets up. 
In other hospital settings without smartphones, an audible alarm would be triggered near the patient's room. All nurses in the interviews emphasized that having the alarm ring on their phone rather than by the patient's door ensured that the patient's nurse would hear the alarm no matter where they were on the unit. This direct communication allowed nurses to have a quick response to urgent situations. This also provided the additional benefit of reducing overhead noise, which is common with traditional bed exit alarms:

In a non-digital hospital, they have overhead paging, and patients are trying to sleep, so they get awakened by the overhead paging.

An additional advantage of the smartphone was its ability to send a notification to a secondary nurse if the primary nurse did not answer the call within a specified time. Furthermore, if there was no response from the secondary nurse, the call would automatically go to the nursing team lead. This process ensured that someone would still be notified and accountable for responding and attending to the patient if the primary nurse was unable to attend to them:

Call bells are answered quickly now, the bed alarm, transfers to [a] secondary nurse - all these really help ensure patient safety.

Nurse-patient relationship Interviews with nursing staff also highlighted improvements to the nurse-patient relationship. Most of the participants stated that because their patient's call goes straight to them rather than to the nursing station, nurses were able to establish a one-to-one relationship with that patient:

You can answer the call and tell the patient, "I am coming; I am just busy," and there's that communication with the patient that you build with them so that they don't wonder why you aren't coming.

\section{Smartphone issues}

One of the major disadvantages reported by the survey participants was the poor call quality and interference on the smartphones. All nurses experienced at least some call quality issue that prevented them from fully using the phones to speak with patients:

I would say it's a good system, but the only problem is [there are] some issues with the [smart] phone: calls drop [and] there's a lot of echo sound that is so bad that you have to hang up and call again because you cannot hear the patient properly. 
A few nurses stated that they preferred to "just pop by" the patient's room after their phone alerts them of a call bell rather than picking up the call through the smartphone. As well, some nurses reported that some patients did not know how to use the phone in the room to speak to the nurse or had trouble increasing the volume.

Additionally, despite acknowledging that the smartphones had increased efficiency, most of the nurses described experiencing a certain degree of stress due to receiving multiple notifications via the smartphone, similar to the stress experienced due to overuse of personal smartphones:

[Smartphones] sometimes cause stress. On some floors, every five to 10 minutes their phone is ringing.

Sometimes I also accidentally hang up the phone because it's touchscreen, so when I put [it] up to my cheek to talk, it hangs up [laughs].

A few of the nurses also stated that when the smartphones were first introduced, they were perceived unfavourably by patients and families. One nurse stated,

I know that when we first got introduced [to] the [smart] phones, some of the patients mistakenly thought it was a cellphone. So there have been comments that were made by patients saying, "Oh, the nurse is on their cellphone when they are supposed to be with me.” But I think now since we are known as a fully digital hospital, the patients who walk through our door ... expect it.

Other issues mentioned were system glitches and poor battery life, which the study participants stated were similar to problems experienced with personal smartphones.

\section{Opportunities for improvement}

Many of the nurses in the interview described several suggestions for further improvement, including adding the option to text interprofessional team members and adding a phone directory to easily reach staff across the hospital. One nurse noted that there were plans to enable the camera feature in the smartphone to be linked to the patient's electronic medical record (EMR). A few of the nurses stated that this enhancement to the smartphone would be particularly useful for capturing the progress of pressure injuries. 


\section{Discussion}

Previous research has only examined the response times of healthcare providers with the use of smartphones in the hospital setting (Hardyman et al. 2013; Patel et al. 2015). The findings from a previous systematic review identified just 55 studies related to stand-alone smartphone-based healthcare technologies, with merely five of these studies being specific to clinical communication (Mosa et al. 2012). The current study contributes to the limited body of knowledge regarding the use of clinical communication technology in healthcare by providing a comprehensive evaluation of integrated hospital smartphone technology from the nurse's perspective.

Similar to the findings from previous studies (Wu et al. 2010) that revealed mobile phone communication in critical care areas facilitated the rapid transfer of information, nursing staff in this study perceived the smartphone as a valuable tool in reducing communication delays. The results from this study were also comparable to previous research that characterized the healthcare system as highly mobile and thus required healthcare professionals to be extensively mobile, with the appropriate technological resources to fully support that mobility (Ammenwerth et al. 2000; Bardram and Bossen 2000; Burdette et al. 2008). In the current study, nurses identified the advantage of the smartphone as providing immediate communication with the interprofessional clinical team and service providers, including porters, regardless of their location on the unit or in the hospital. Study participants said a key benefit of the smartphone was convenience and improved efficiency, based on a more timely exchange of information, which is consistent with previous research findings (Mobasheri et al. 2015).

This study also supports the proposition from previous research that suggests stand-alone smartphone applications are limited in their usefulness to healthcare professionals and must be integrated with other health information systems to enhance patient care (Mosa et al. 2012). The findings from the current study revealed that nurses viewed the integration of the smartphone with the patient call-bell system as integral to delivering safe care. Nurses placed a high value on the tone-varied alerts that are received directly through the smartphone and serve as a patient needs prioritization tool - for example, the alarm for preventing a patient fall will sound on the smartphone as a top priority.

The results of this study revealed that nurses perceived that smartphone technology improved relationships with patients because calls are received directly, which fosters continuity and comprehensiveness of care delivery. Similar results were found in a previous study, whereby nurses and physicians reported little support 
for the notion that smartphone technology "gets in the way of care" (Moore and Jayewardene 2014). These findings suggest that nurses and other healthcare providers may be more primed than in the past to accept the use of technology as a means to enhance care delivery without compromising the therapeutic relationship.

In addition, the findings from this study were similar to previous research results in which some level of discomfort existed among healthcare providers with using smartphone technology in the presence of patients (Moore and Jayewardene 2014). Nurses in the current study perceived that patients perceived smartphones unfavourably because of misunderstandings about its use for personal versus clinical communication and care delivery. This suggests that hospitals proposing to introduce smartphone technology may need to ensure that patients and families are well educated about the clinical use and functionality of communication information technology.

The smartphone integration at HRH permits nurses to receive information from a variety of digital devices. Currently, the smartphone at HRH serves primarily as a one-way information source for nurses. As nurses in this study have suggested, the ability to text interprofessional team members may support better and faster information exchange between clinicians. However, text communications between healthcare providers pose some risks. First, all patient information must be documented in the EMR to ensure that standards and legal requirements are met in relation to patient care delivery. Second, clear and verifiable mechanisms are required for receiving and confirming medical orders to avoid errors. To become a tool for information exchange between nurses and other clinicians, the smartphone would need to be interoperable with the EMR so that every aspect of care delivery could be digitally recorded, including clinical photographs. $\mathrm{HRH}$ is exploring these opportunities to further leverage the use of smartphone technology and maximize the potential for streamlined, ultra-safe care delivery of the highest quality.

Further reflecting on the issue of communication in the fully digital environment, the integration of smartphone technology may provide a crucial connection between healthcare team members. Anecdotally, nurses and other clinicians have commented that with the introduction of computerized provider order entry (CPOE), in which medical orders are completed electronically and automatically sent to nurses or other healthcare providers, there are fewer opportunities to communicate among the team. The introduction of smartphone technology has fostered the opportunity to communicate and reconnect as members of the 
healthcare team. Organizations at the initial stages of digitalization may want to consider the integration of smartphone technology alongside CPOE to reduce the likelihood that communications between the interprofessional team are disrupted.

Nurses in this study revealed their experience of being stressed because of receiving multiple notifications via the smartphone. This is an important finding but not surprising given the challenges associated with achieving a balance between alarm support and alarm fatigue. Healthcare organizations will need to be conscious of this phenomenon when implementing technology and strive to create a balance.

Overall, research participants perceived the clinical use of the smartphone and its integrated features in a very positive light. Issues such as patients' lack of knowledge regarding how to use the call system, insufficient sound quality and poor battery life were similar to problems identified in previous studies (Mosa et al. 2012). Considering the multiple benefits of smartphone technology identified by nurse participants in this study, including time management, convenience, prioritization, patient safety and enhancement of the nurse-patient relationship, other healthcare organizations may be persuaded and supported to implement mHealth technology.

\section{Limitations}

This study has some limitations, which must be addressed. First, respondent selection was limited due to nurses' busy schedules; therefore, this presents a degree of non-response bias. Next, the data were derived from a single hospital site, limiting the generalizability to other hospital settings, specifically smaller hospitals, which may not have the same needs or obstacles as larger hospitals. Additionally, the study outcome was limited to nurse perceptions of smartphone technology, and patient perceptions were not obtained. Finally, face-to-face interviews may lead to social desirability bias; however, interviews were conducted by the research coordinator to minimize any influence on the participants' responses.

\section{Conclusion}

The integration of smartphone technology with the patient call-bell system provides the opportunity to enhance patient safety by supporting nurses' ability to directly communicate and prioritize care delivery. Aside from the potential for harm reduction, the findings from this study suggest further benefits of integrated communication information technology, such as improved nurse-patient relationship, time management and convenience. Integrated smartphones have the prospect of changing the way nursing care is provided. The findings from this study suggest that smartphones can play an important role in enhancing the delivery of high-quality care. 


\section{Acknowledgements}

We are grateful to Alisha Aggarwal for her help in recruiting participants, and we thank all the nursing staff who participated in the study.

\section{Correspondence may be directed to: Dr. Vanessa Burkoski, Humber River Hospital, 1235 Wilson Avenue, Toronto, ON, M3M 0B2; email: vburkoski@hrh.ca.}

\section{References}

Ammenwerth, E., A. Buchauer, B. Bludau and R. Haux. 2000. "Mobile Information and Communication Tools in the Hospital." International Journal of Medical Informatics 57(1): 21-40.

Bardram, J.E. and C. Bossen. 2005. "Mobility Work: The Spatial Dimension of Collaboration at a Hospital." Computer Supported Cooperative Work 14(2): 131-60.

Burdette, S.D., T.E. Herchline and R. Oehler. 2008. "Surfing the Web: Practicing Medicine in a Technological Age: Using Smartphones in Clinical Practice." Clinical Infectious Diseases 47(1): $117-22$.

Doswell, W.M., B. Braxter, A.D. Dabbs, W. Nilsen and M.L. Klem. 2013. "mHealth: Technology for Nursing Practice, Education, and Research." Journal of Nursing Education and Practice 3(10): 99-109.

Galinato, J., M. Montie, L. Patak and M. Titler. 2015. "Perspectives of Nurses and Patients on Call Light Technology." Computers, Informatics, Nursing 33(8):359-67.

Hardyman, W., A. Bullock, A. Brown, S. Carter-Ingram and M. Stacey. 2013. "Mobile Technology Supporting Trainee Doctors' Workplace Learning and Patient Care: An Evaluation." BMC Medical Education 13(1): 6 .

Mobasheri, M.H., D. King, M. Johnston, S. Gautama, S. Purkayastha and A. Darzi. 2015. "The Ownership and Clinical Use of Smartphones by Doctors and Nurses in the UK: A Multicentre Survey Study." BMJ Innovations 1: 174-81.

Moore, S. and D. Jayewardene. 2014. “The Use of Smartphones in Clinical Practice." Nursing Management-UK 21(4): 18-22.

Mosa, A.S., I. Yoo and L. Sheets. 2012. "A Systematic Review of Healthcare Applications for Smartphones." BMC Medical Informatics and Decision Making 12(1): 67.

Patel, R.K., A.E. Sayers, N.L. Patrick, K. Hughes, J. Armitage and I.A. Hunter. 2015. "A UK Perspective on Smartphone Use amongst Doctors within the Surgical Profession." Annals of Medicine and Surgery 4(2): 107-12.

Prakash, V., C. Koczmara, P. Savage, K. Trip, J. Stewart, T. McCurdie et al. 2014. “Mitigating Errors Caused by Interruptions during Medication Verification and Administration: Interventions in a Simulated Ambulatory Chemotherapy Setting." BMJ Quality \& Safety 23(11): 884-92. doi:10.1136/ bmjqs-2013-002484.

Redding, D.A. and S. Robinson. 2009. “Interruptions and Geographic Challenges to Nurses' Cognitive Workload." Journal of Nursing Care Quality 24(3): 194-200.

Tomietto, M., A. Sartor, E. Mazzocoli and A. Palese. 2012. "Paradoxical Effects of a Hospital-Based, Multi-Intervention Programme Aimed at Reducing Medication Round Interruptions." Journal of Nursing Management 20: 335-43.

Westbrook, J.I., A. Woods, M.I. Rob, W.T. Dunsmuir and R.O. Day. 2010. “Association of Interruptions with an Increased Risk and Severity of Medication Administration Errors." Archives of Internal Medicine 170(8): 683-90.

Whitlow, M.L., E. Drake, D. Tullmann, G. Hoke and D. Barth. 2014. "Bringing Technology to the Bedside: Using Smartphones to Improve Interprofessional Communication. Computers, Informatics, Nursing 32(7): 305-11.

Wu, R.C., D. Morra, S. Quan, S. Lai, S. Zanjani, H. Abrams et al. 2010. "The Use of Smartphones for Clinical Communication on Internal Medicine Wards." Journal of Hospital Medicine 5(9): 553-59. 


\section{$\mathbf{H}_{\substack{\text { Hosmegra neve } \\ \text { Hosith }}}^{+}$}

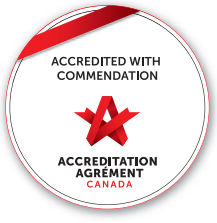

DOO
$\mathbf{D}$ BEST PRACTICE
SPOTLIGHT
ORGANIZATION
C A N A D A A
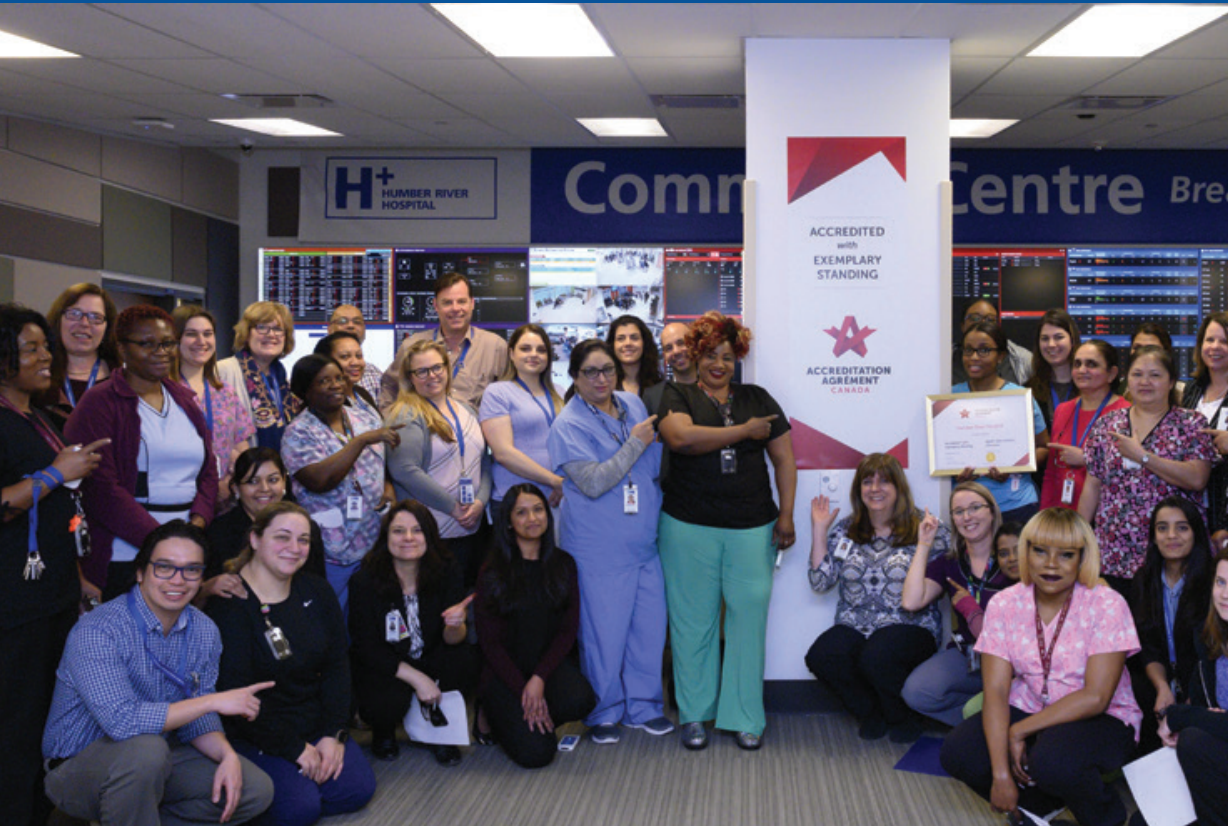

ACCREDITED

entre Breakthrough Intelligence

STEMPLARY

* cactorernon
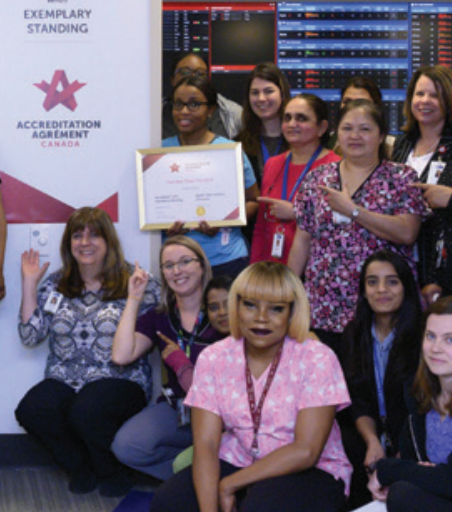

$\sin 2$
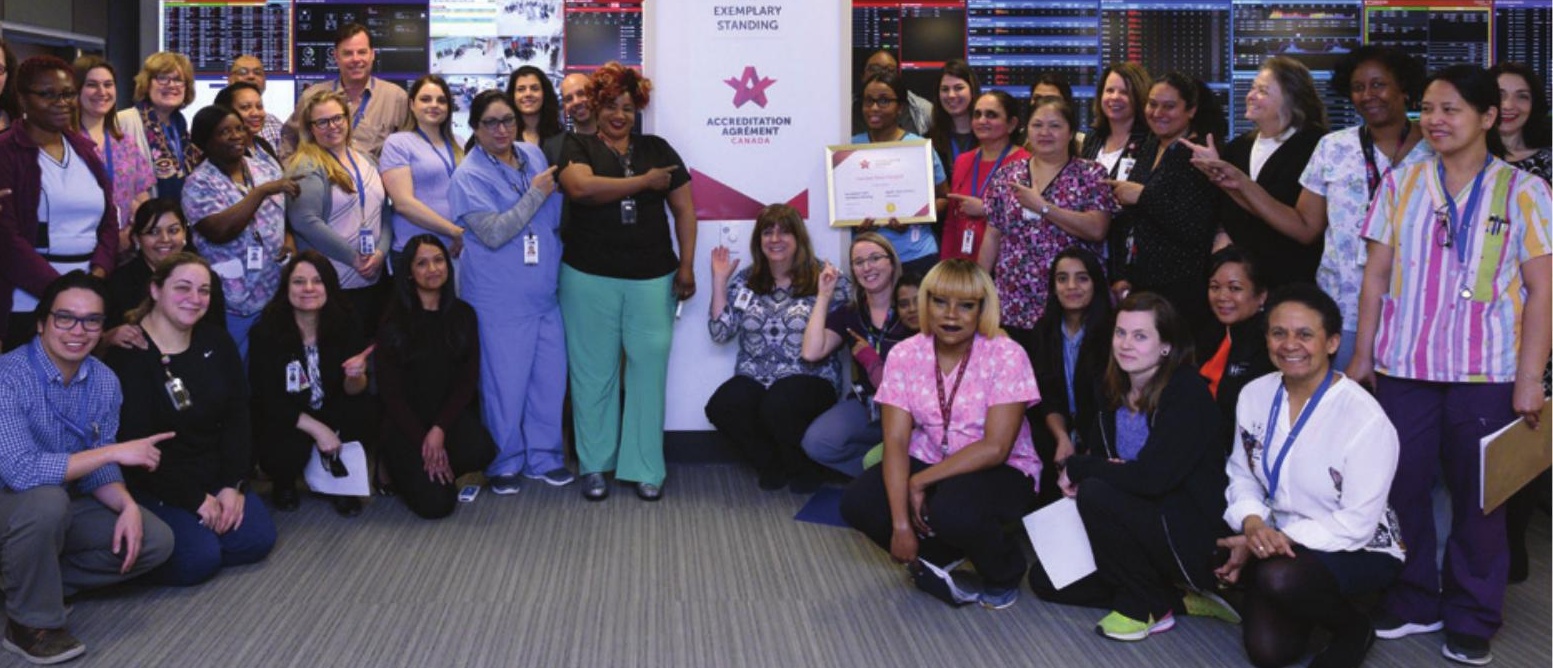

\section{Leading technology \& innovation integration in nursing}




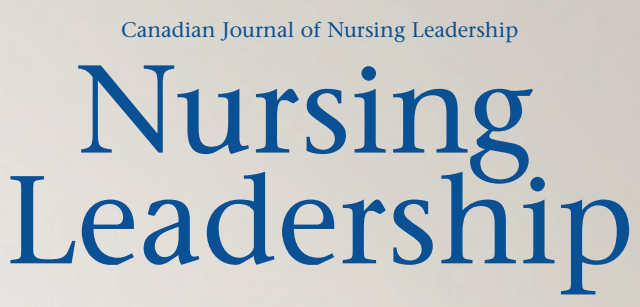

Leadership in Nursing Management, Practice, Education \& Research

NURSING LEADERSHIP - PERSPECTIVES AND INSIGHTS

Building Healthcare Leadership Capacity: Strategy, Insights and Reflections 10

The Role of Education in Developing Leadership in Nurses

Engaging Nurses in Future Management Careers: Perspectives on Leadership and Management Competency Development through an Internship Initiative 36

Black Nurse Leaders in the Canadian Healthcare System 50

Politics • Policy • Theory • Innovation

Us

ACEN
ACDSI

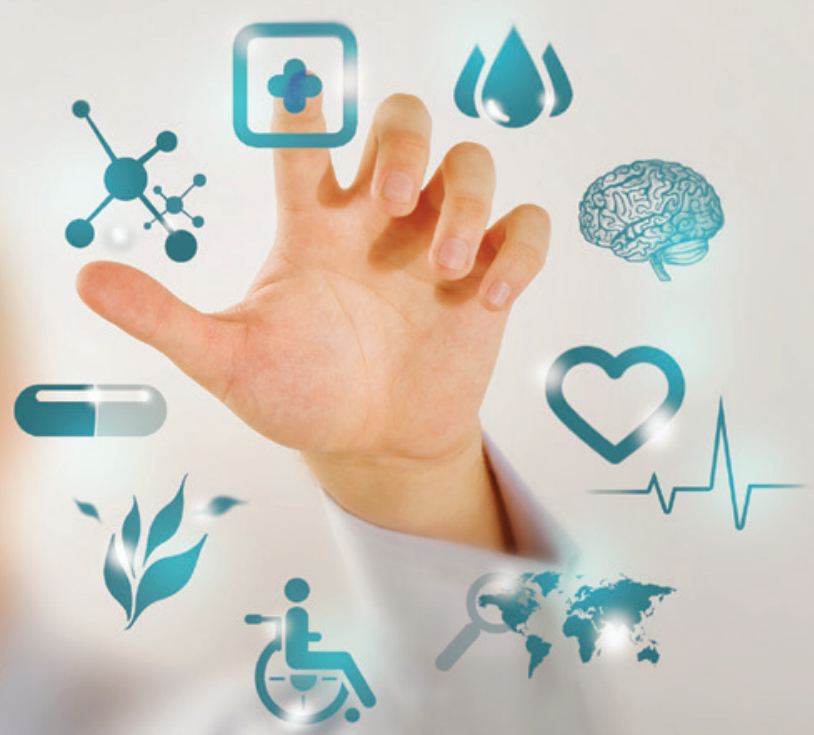

Covering politics, policy, theory and innovations that contribute to leadership in nursing administration, practice, teaching and research. 